\title{
Friction coefficient measurements of silencers on specialized duct tract
}

\author{
Stanislav Sehnalek ${ }^{1, a}$, Pavel Drabek ${ }^{1}$ and Martin Zalesak ${ }^{1}$ \\ ${ }^{1}$ Tomas Bata University in Zlin, Faculty of Applied Informatics, Department of Automation and Control Engineering, Nad Stranemi \\ 4511, Zlin, Czech Republic
}

\begin{abstract}
This article describes test methods on air duct track in Laboratory of Environmental Engineering. It focuses on measurement of silencer parameter like is pressure loss coefficient. Firstly, the paper describe the measurement apparatus with description of calculation method by standard ISO 7235 and energy equation. Then the paper presents three ways how to accomplish measurement because such way is not covered by procedure in standard. Then follows the evaluation of results of measurements on three types of silencer designed for HVAC applications. The article is concluded with discussion over measured data with outline for further research.
\end{abstract}

\section{Introduction}

Aerodynamic properties of air is in the scope of interest of researchers for more than century. The air flow parameters was investigated by Hagen, Reyleigh, Reynolds [1], followed up by Prandtl, Moody [2], Colebrook [3], Von Karman [4] and many others. [5] The knowledge of concept properties concerning HVAC components is a prerequisite for successful design technique. In the draft of ventilation ducts the pressure loss is an important parameter to know based on different velocities of transported air. Determination of parameters for specific HVAC devices are in scope of specialized laboratories. Laboratory of such capabilities is maintained by authors of this article at Tomas Bata University in Zlin, Faculty of Applied Informatics and is involved in this paper. Special interest in actual development is focused in acoustic silencers for mounting in the ducts which are important element within HVAC systems. With the silencers there are two main parameters which should be taken care off, first one is a sound attenuation and second one are pressure losses. It is regrettable that those parameters are in opposition to each other. In facility of the authors the Laboratory of Environmental Engineering it is possible to test the silencers for both parameters and thus combine both parameters for better performances. In presented paper, the focus is on measurement methods described by standard ISO 7235 [6] and comparison of results obtained by this standard with analytical calculations with different approach. Mainly, this standard lacks any details how to accomplish such measurement of friction losses. Investigation of such procedure is the subject of presented paper, where three possible ways hot to executed measurement of silencers are compared.

In the presented article is firstly described the methods of measurement with the test track, with the descrip- tion of calculation for losses by standard and alternative method. Above mentioned is linked with the description of measurement method with characterization of used samples. Then the measured results are described, followed by discussion. Article is wrapped up by conclusion of used methods and samples.

\section{Methods}

There is a possibility to measure minor loss coefficient of any equipment determined for installation inside a duct. For this measurements is used multiple-nozzle chamber to resolve flow rate through the duct configuration. There is taping mount on ducts for measurement of static pressure before and after surveilled object. Such measurements and consequential calculations are in scope of this article. Firstly, duct track will be covered followed by description of two options for determination of minor loss coefficient. At first by ISO 7235 [6] and subsequently by hydraulic

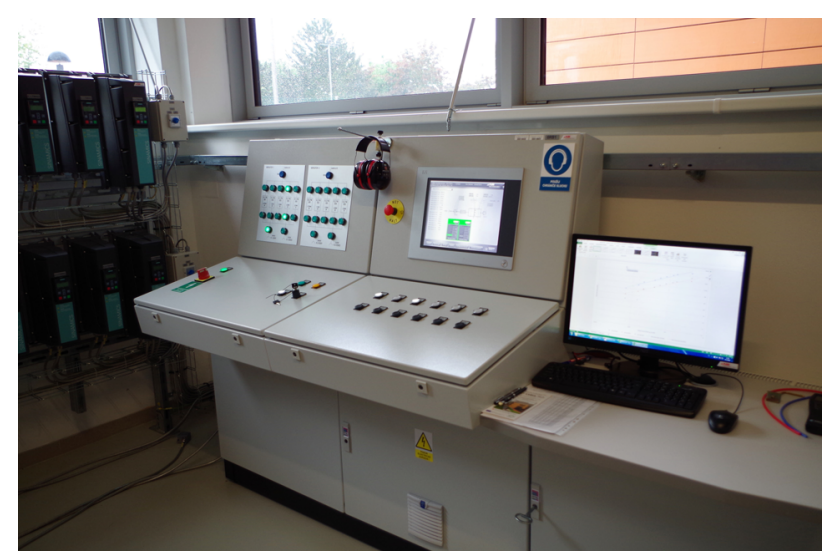

Figure 1. Controlling board for duct tract

\footnotetext{
${ }^{\mathrm{a}}$ Corresponding author sehnalek@fai.utb.cz
} 


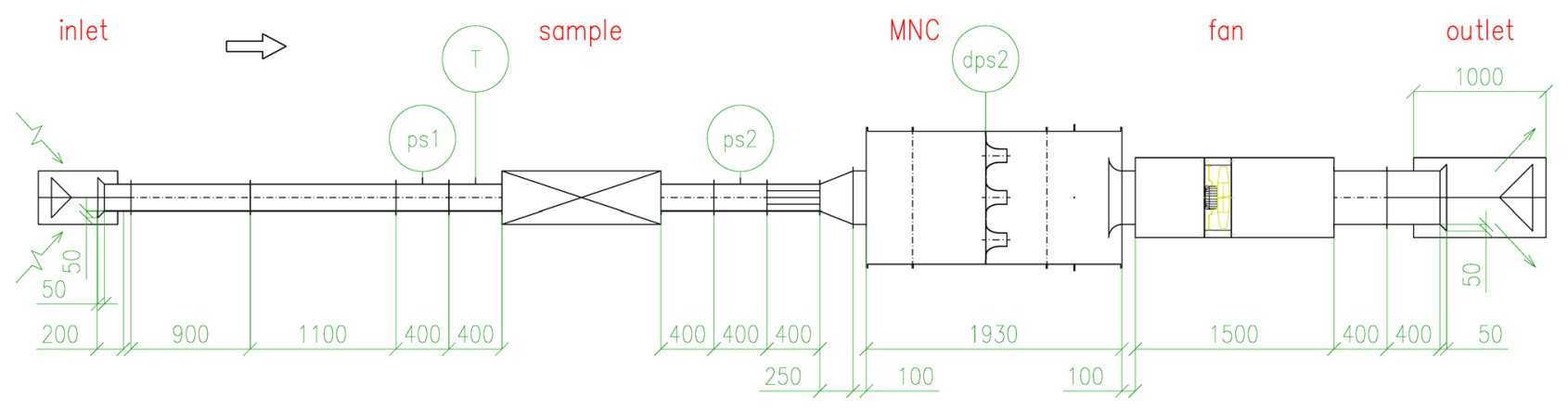

Figure 2. Track for measurement of friction loss coefficient

equation. At the end of this section will be a brief description of silencers which was used as samples.

\subsection{Fan track}

There are two available dimensions for the track. The diameters are $200 \mathrm{~mm}$ and $400 \mathrm{~mm}$. For each dimension there are available several type of duct which could be connected with each other. This function lead to many variations for measurements. At the track could be done measurements of fan performance curve, loss coefficient, acoustic parameters, leakage and other measurements. All components of track are made in accordance with International standard ISO 5801. Controlling system of track is handled by PLC with touch panel including visualization. All measured data are periodically saved to local FTP server in form of CSV file. Whole controlling board is depicted on Figure 1. On the left could be seen frequency converters for fans, next to it is buttons for switching circuit breaker, then is touch panel with visualization for setting experiment and on the right is computer unit. For purpose of measurement was used an automated excel sheet, where the manually loaded data and all calculations are ensued by graphical output. The data collection is started after two minutes phase within is stabilized conditions and are collected for one minute every half second. This means that for each point were collected 120 values which are averaged afterwards.

Measurement track is depicted in Figure 2. The whole length without the sample is more than $9 m$ long. It consist of inlet, ducts, sample, ducts, straightener, size extension, multiple-nozzle chamber (MNC), fan, duct and outlet. Ducts before MNC are of diameter $200 \mathrm{~mm}$, tract after $\mathrm{MNC}$ are of dimension $400 \mathrm{~mm}$.

\subsection{Minor loss coefficient by CSN EN ISO 7235}

In the following text will be described method to measure minor loss coefficient by international standard ISO 7235. [6] This norm is mainly used for duct silencers and describes the measuring of sound attenuation as well as the measurement of minor loss coefficient. Evaluation of friction coefficient is done by subtracting the pressure difference at substituted duct from static pressure difference of silencer. All computational steps are described by following equations.

$$
\Delta p_{t o t}=p_{s 1(I)}-p_{s 1(I I)}
$$

Where is $\Delta p_{\text {tot }}$ total pressure

$p_{s 1(I)} \quad$ pressure drop at measured device

$p_{s 1(I I)} \quad$ pressure drop of substituted duct

The value $p_{s 1(I)}$ is the difference of static pressure between the measured sample. The value $p_{s 1(I I)}$ is same as before only performed on substituted duct. There is also included measurement between static pressure before sample and atmospheric pressure $p_{s 1(a)}$ only for graphical representation of results. In the following equations 2,3 can be seen how to calculate dynamic pressure and determine density of inlet air.

$$
p_{d}=\frac{\varrho_{i n}}{2} v^{2}=\frac{\varrho_{i n}}{2}\left(\frac{q_{v}}{A}\right)^{2}
$$

Where is $p_{d}$ inlet dynamic pressure

$$
v \quad \text { flow velocity }
$$

$A$ area at the point of measurement of static pressure

$\varrho_{\text {in }} \quad$ density of inlet air $\quad\left[\frac{\mathrm{kg}}{\mathrm{m}^{3}}\right]$

$q_{v} \quad$ volumetric flow rate $\quad\left[\frac{\mathrm{m}^{3}}{\mathrm{~s}}\right]$

$$
\varrho_{\text {in }}=\frac{p_{s 1}+p_{a}}{R\left(\theta_{\text {in }}+273\right)}
$$
Where is $R \quad$ gas constant 287 $\left[\frac{\mathrm{N} \cdot \mathrm{m}}{\mathrm{kg} \cdot \mathrm{K}}\right]$
$\theta_{\text {in }}$ temperature of air $\quad\left[{ }^{\circ} \mathrm{C}\right]$ before sample

The coefficient of the total pressure loss $\xi$ averaging over a range of flow rate is calculated from the equation (4).

$$
\xi_{\text {iso }}=\frac{\Delta p_{t o t}}{p_{d}}=\frac{\Delta p_{t o t}}{\frac{\rho}{2}\left(\frac{q_{V}}{A}\right)^{2}}
$$

Where is $\xi_{\text {iso }}$ minor loss coefficient by ISO 7235

A area of duct $\left[m^{2}\right]$ 

(5).

Mean loss coefficient is then calculated from equation

$$
\xi_{i s o, m}=\frac{1}{N} \sum_{j=1}^{N} \frac{p_{s 1(I) j}}{p_{d j}}-\frac{1}{M} \sum_{k=1}^{M} \frac{p_{s 1(I I) k}}{p_{d k}}
$$

Where is $\quad N \quad \begin{aligned} & \text { number of measured } \\ & \text { points of silencer }\end{aligned} \quad[-]$

\subsection{Minor loss coefficient from Bernoulli equation}

Other option could be the determination of $\xi$ from energy equation which is summarized in (6), which is known also as Bernoulli Equation.

$$
p_{1}+\frac{\rho v_{1}^{2}}{2}+h_{1} \rho g=p_{2}+\frac{\rho v_{2}^{2}}{2}+h_{2} \rho g+\Delta p_{\text {loss }}
$$

$\begin{array}{llll}\text { Where is } & p & \text { static pressure } & {[\mathrm{Pa}]} \\ & h & \text { elevation height } & {[\mathrm{m}]} \\ g & \text { acceleration of gravity } & {\left[\frac{\mathrm{m}}{\mathrm{s}^{2}}\right]} \\ & \Delta p_{\text {loss }} & \text { pressure loss } & {[\mathrm{Pa}]}\end{array}$

The focus is now on $\Delta p_{\text {loss }}$ which is specified by equation (7).

$$
\Delta p_{\text {loss }}=\Delta p_{\lambda}+\Delta p_{\xi}
$$

Where is $\begin{array}{rrr}\Delta p_{\lambda} & \text { major loss } & {[\mathrm{Pa}]} \\ \Delta p_{\xi} & \text { minor loss } & {[\mathrm{Pa}]}\end{array}$

The equation (8) summarizes the Darcy-Weisbach Equation for calculation of major loss and in equation (9) is noted the calculation of minor loss .

$$
\Delta p_{\lambda}=\lambda \frac{l}{d} p_{d}=\lambda \frac{l}{d} \frac{\rho}{2} v^{2}=\lambda \frac{l}{d} \frac{\rho}{2}\left(\frac{q_{V}}{A}\right)^{2}
$$

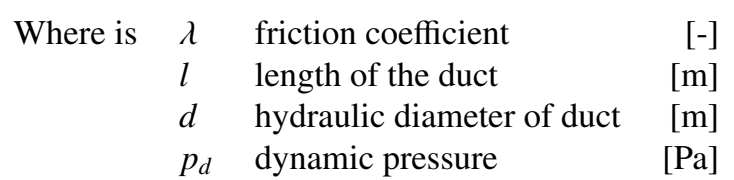

Minor loss coefficient also, known as friction coefficient, is dependent only on Reynolds number for laminar flow. For turbulent flow matters the friction coefficient function of Reynold number as well as of roughness of the duct. In 1937 Colebrook and White presented experiment of friction coefficient in roughness duct and established Colebrook-White formula [7]. There are at least three implicit formulas known by today literature. This article will present only best known of them; thus authors of this article compared results with all of implicit equations and there have been negligible aberration in results. Used equation is formulated in (10) which presented Collebrook in 1939.[3][8] There have been many attempts to provide explicit form in the past. Mainly because iterative process of implicit equation was complicated in time where there haven't been calculators. It would be possible to use so called Moody chart, which was laid out by prof Moody in 1944 as estimation from Colebrook-White formula. This chart made it easy to estimate friction coefficient and was extensively used by engineers for more than half century. [2][8] This changed with arrival of electronic chips and computers where was no problem to use iterative process. [8]

$$
\Delta p_{\xi}=\xi_{b e} p_{d}=\xi_{b e} \frac{\rho}{2} v^{2}=\xi_{b e} \frac{\rho}{2}\left(\frac{q_{V}}{A}\right)^{2}
$$

Where is $\xi_{b e}$ minor loss coefficient [-] by Bernoulli equation

$$
\frac{1}{\sqrt{\lambda}}=-2 \log \left(\frac{2,51}{\operatorname{Re} \sqrt{\lambda}}+\frac{\varepsilon}{3,7}\right)
$$

Where is $R e$ Reynolds number

$\varepsilon \quad$ Roughness coefficient of duct [m]

So called Reynold number was introduced in 1883 by Osborne Reynolds and it is transcribed in (11). As Reynolds number came to known by usage of Sommerfeld and Prandtl at the beginning of the last century. [1][5]

$$
R e=\frac{v d}{v}=\frac{\rho v d}{\mu}
$$

Where is $v$ kinematic viscosity of the fluid $\left[\frac{\mathrm{m}^{2}}{\mathrm{~s}}\right]$ $\mu$ dynamic viscosity of the fluid $\left[\frac{N_{s}}{\mathrm{~m}^{2}}\right]$

From above equation (7) could be determined minor loss coefficient $\xi_{b e}$ by simple mathematical extraction and it is equal to (12). Also (13) could be used, particularly because there is unknown roughness coefficient for the silencers so $\lambda$ should be neglected.

$$
\begin{gathered}
\xi_{b e, \lambda}=\frac{2 \Delta p_{\text {loss }}}{\rho v^{2}}-\lambda \frac{l}{d} \\
\xi_{b e}=\frac{2 \Delta p_{\text {loss }}}{\rho v^{2}}
\end{gathered}
$$

\subsection{Measurement practice}

Measurement was performed in accordance with ISO 7235 and flow determination ISO 5801. [6][9] In the standards there is specified tapping to obtain average static pressure. Before tapping should be straight duct in length at least $5 d$ or two meters long, depends which value is higher. It is also stated, that position of tapping should be $1,5 d$ from entrance to tested object as well as at exit from it. Measurement of temperature inside the duct is specified as $2 d$ in front of the tested specimen. [6]

In standard ISO 7235 is not specified method of reduction of flow, nor is stated if measurement should be done in-front or behind the fan. So in this articlethe measurement was performed at inlet side of fan, outlet side will be subject of further interest. Due to the nature of measurement tract it was possible to perceived three types of flow reduction. Description of those three category and naming is as follows: 


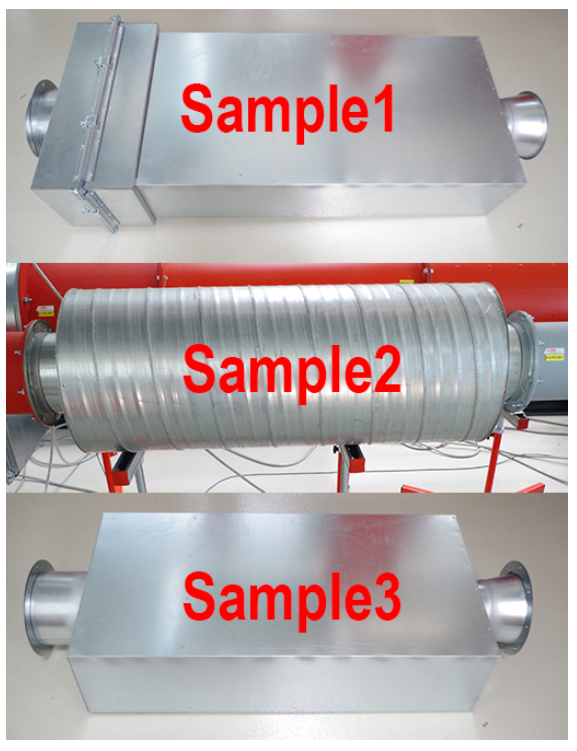

Figure 3. Silencers used as samples

- InRedu - Reduction of flow and static pressure is done by throttling at inlet duct.

- OutRedu - Reduction of flow and static pressure is done by throttling at outlet duct. Decreasing of flow was done based on FanRedu values of flow for comparison of results.

- FanRedu - Reduction of flow and static pressure is done by decreasing power of ventilator (this method could be persecuted from $100 \%$ to $20 \%$ of ventilator power, where selected steps for fan was by $10 \%$ ).

\subsection{Silencer specimens}

Measurement was done on three types of silencers and they can be seen in Figure 3. Description of those samples is as follows:

- Sample1 - This silencer is most complicated one, it has square outside shape with round inside silencing part. Its outlet is made from 9 small connections of diameter $80 \mathrm{~mm}$. For purpose of measurement was created reduction chamber with $0,2 m$ diameter outlet to fit to the measurement tract. The whole length is $1,45 \mathrm{~m}$ and outer dimensions are $250 \times 500 \mathrm{~mm}$.

- Sample2 - This silencer is round with centre body. Its length is $1,2 \mathrm{~m}$ and outer diameter is $0,4 \mathrm{~m}$.

- Sample3 - The last silencer is same as first one, but outlet part with 9 connections is removed and it is directly connected to $0,2 m$ fitting. The length is in this case $1,3 m$.

\section{Results}

The following section contains results and findings during the performed measurements. Firstly will be mentioned method InRedu and its results. This will be forwarded with results of OutRedu and FanRedu methods for all samples.

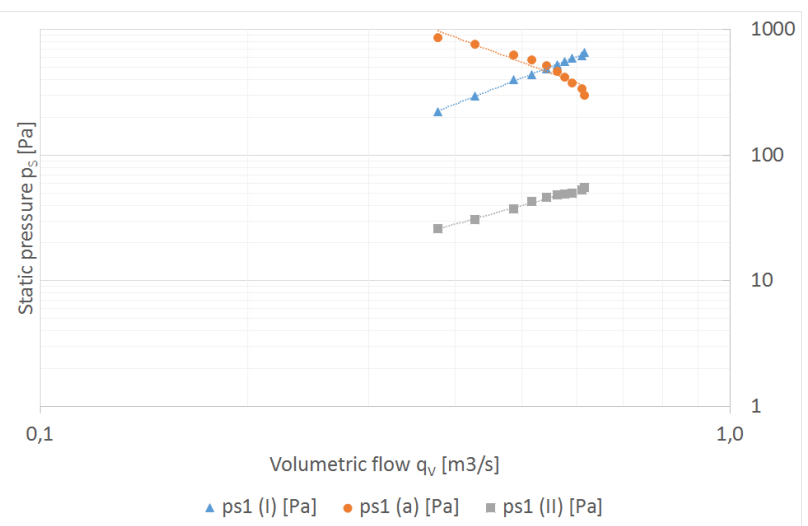

Figure 4. Log-log plot of inlet reduction for Sample1

All the presented graphs are in log-log scale. This section will conclude with comparison of two types of calculation which was described in Methods section and introduction to option for backward finding of roughness coefficient.

\subsection{Results for InRedu method}

This method was tested only on Sample1 and is depicted in Figure 4. The method could be hardly used for appropriate presentation of results, nevertheless the friction coefficient was in the end similar as in other methods which will be presented.

\subsection{Results forOutRedu and FanRedu methods}

Pressure drop and flow through the duct with method OutRedu and FanRedu are depicted in Figures 5, 6 and 7. Difficulty $d u$ ring m e asurement $h$ a ppened $w$ hen $t h$ ere $w$ a s a necessity to measure substituted duct with the FanRedu method. When was executed measurement of substituted duct it should proceed with same flow as s a mple which was at the beginning always around $60 \%$ with inequivalent steps. Reason of this is due to steep losses of supplementary duct. This is reflected by the substituted curve, which has less points because fan can not go less than $20 \%$ of power. From the figures $\mathrm{c}$ ould b e s een $\mathrm{t}$ hat both methods are similar and could be commuted. This fact is also digested in Table 1, where could be seen differences in methods by friction coefficient. Most significant difference in methods was $\xi=0,054$ and occurred with Sample2, which is careless amount.

Table 1. Comparison of methods with calculation by CSN

\begin{tabular}{cccc} 
Sample & Method & $\xi_{c s n}[-]$ & diff [-] \\
\hline \hline \multirow{2}{*}{ Sample1 } & OutRedu & 2,851 & \multirow{2}{*}{0,044} \\
& FanRedu & 2,808 & \\
\hline \multirow{2}{*}{ Sample2 } & OutRedu & 2,598 & \multirow{2}{*}{0,054} \\
& FanRedu & 2,544 & \\
\hline \multirow{2}{*}{ Sample3 } & OutRedu & 0,924 & \multirow{2}{*}{0,014} \\
& FanRedu & 0,911 &
\end{tabular}




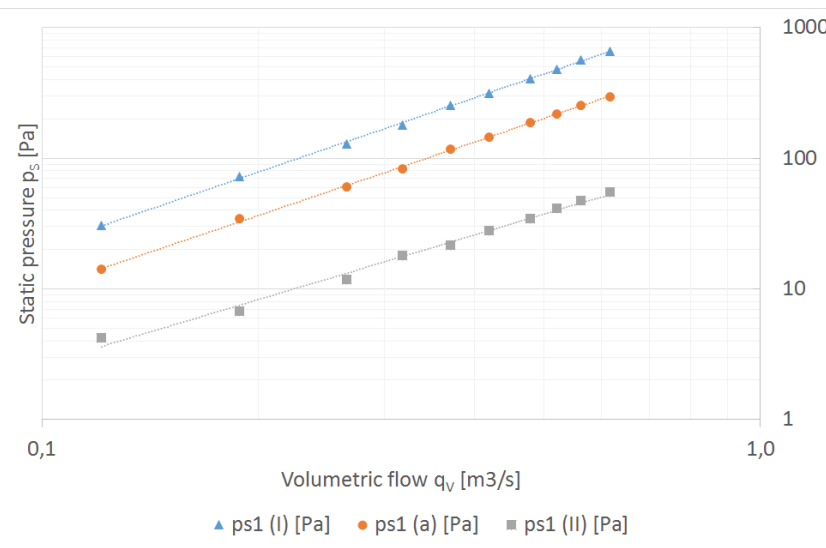

(a) OutRedu

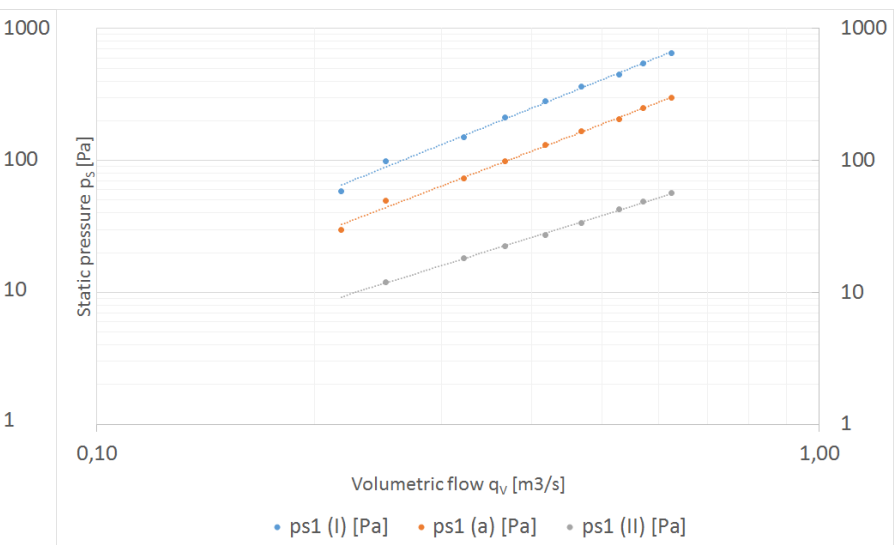

(b) FanRedu

Figure 5. Log-log plots for Sample1

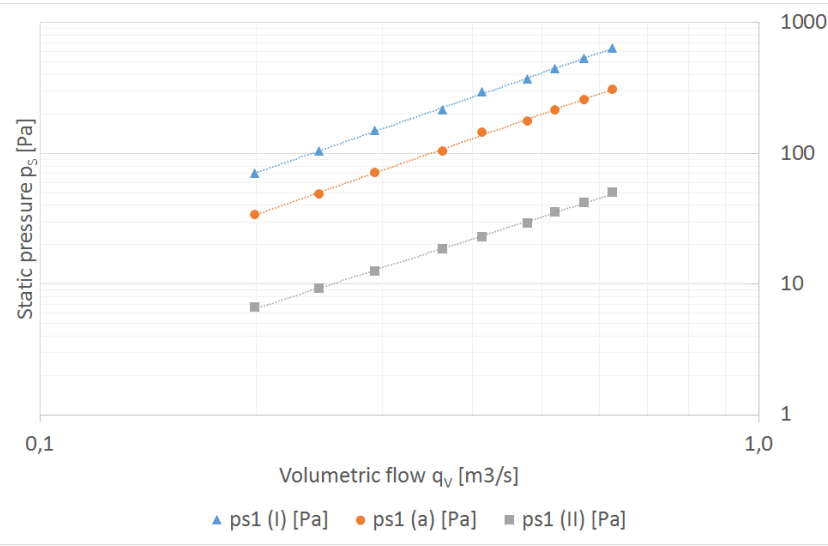

(a) OutRedu

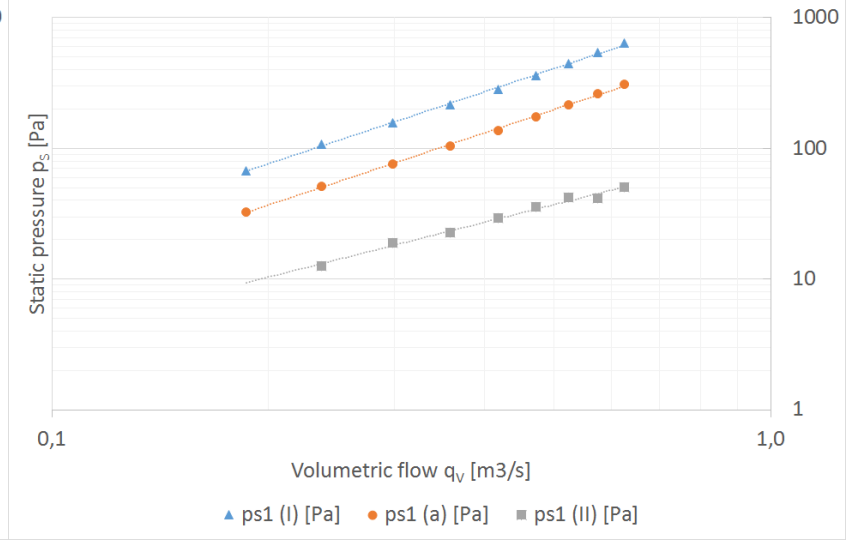

(b) FanRedu

Figure 6. $\log -\log$ plots for Sample2

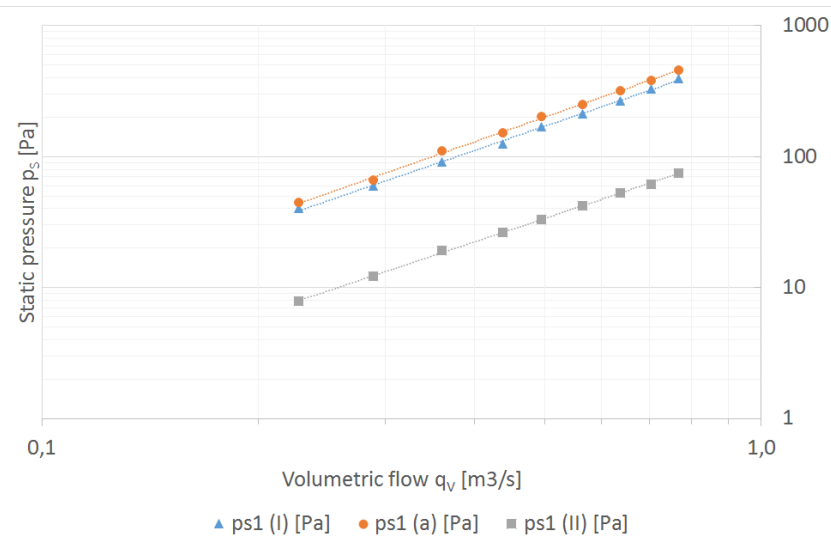

(a) OutRedu

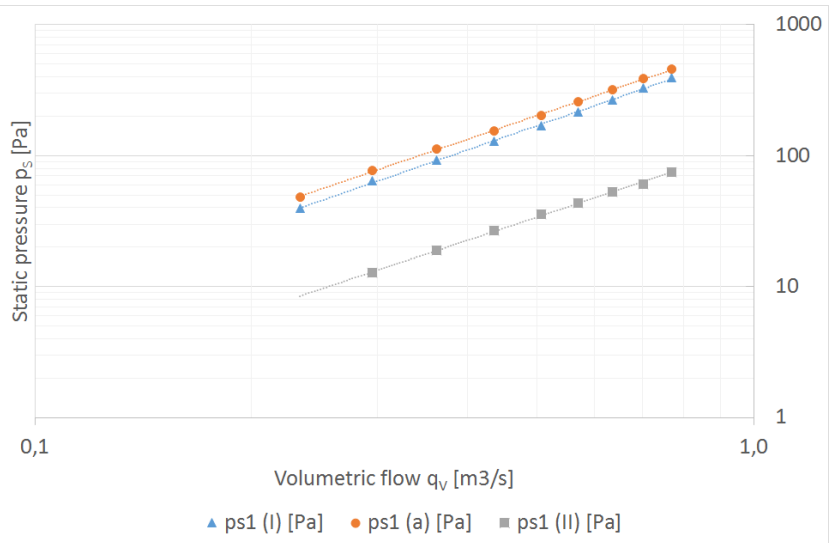

(b) FanRedu

Figure 7. Log-log plots for Sample3

From the Table 2 could be seen that all flows were in turbulent region based on Reynolds numbers.
Results concerning calculation of $\xi_{b e}$ are written in Table 3, where the most significant difference with Sample1 
Table 2. Minimal and maximal Reynolds number for each sample and method

\begin{tabular}{clcc} 
Sample & Method & $\operatorname{Re} \min [-]$ & $\operatorname{Re~max~[-]~}$ \\
\hline \hline \multirow{2}{*}{ Sample1 } & OutRedu & 49730 & 254576 \\
& FanRedu & 89678 & 257955 \\
\hline \multirow{2}{*}{ Sample2 } & OutRedu & 81719 & 258050 \\
& FanRedu & 76512 & 258035 \\
\hline \multirow{2}{*}{ Sample3 } & OutRedu & 93414 & 316163 \\
& FanRedu & 95922 & 317042
\end{tabular}

is evident. The inequality with standard method is due to fact that method measured by subtracting pressure with substituted duct could include inaccuracy.

Table 3. Comparison of methods with calculation by BE

\begin{tabular}{cccc} 
Sample & Method & $\xi_{b e}[-]$ & diff $[-]$ \\
\hline \hline \multirow{2}{*}{ Sample1 } & OutRedu & 3,099 & \multirow{2}{*}{0,117} \\
& FanRedu & 3,216 & \\
\hline \multirow{2}{*}{ Sample2 } & OutRedu & 2,834 & \multirow{2}{*}{0,042} \\
& FanRedu & 2,876 & \\
\hline \multirow{2}{*}{ Sample3 } & OutRedu & 1,154 & \multirow{2}{*}{0,007} \\
& FanRedu & 1,147 &
\end{tabular}

In the Table 5 are compared two ways of calculation of $\xi$, namely equation (5) with (13). The difference is fluctuating around 0,3 . This diversity is mainly because in $\xi_{c s n}$ was taken total pressure subtracted by pressure of substituted duct as for opposition is taken only total pressure of sample for $\xi_{b e}$.

There are significant differences of coefficients within the used method and samples. This is caused basically because there is difference in $\xi$ calculated by both methods.

Table 4 summarize all friction loss coefficients. First line is used sample and method, thus S1-OR for Sample1 OutRedu method and so on. In some cases missing coefficients, this is due to nature of method where was low limit reached in different points. It is obvious that minor loss coefficient is independent on velocity inside the duct, this fact is valid only for velocity from 3 to $24 \frac{\mathrm{m}}{\mathrm{s}}$. Thus in this

Table 4. Match of loss coefficient for samples by method

\begin{tabular}{cccccc} 
S1-OR & S1-FR & S2-OR & S2-FR & S2-OR & S3-FR \\
\hline \hline 2,6570 & 2,5516 & 2,5070 & 2,5112 & 0,8982 & 0,8987 \\
2,7585 & 2,5658 & 2,5255 & 2,5225 & 0,9055 & 0,9030 \\
2,7043 & 2,4402 & 2,5405 & 2,4592 & 0,8845 & 0,8917 \\
2,7276 & 2,5009 & 2,5066 & 2,4307 & 0,8919 & 0,8848 \\
2,7509 & 2,4317 & 2,7151 & 2,4446 & 0,9330 & 0,8731 \\
2,8609 & 2,3580 & 2,5236 & 2,5303 & 0,8695 & 0,8971 \\
2,7070 & 2,1497 & 2,7128 & 2,6119 & 0,9272 & 0,9442 \\
2,7780 & 2,3227 & 2,6556 & 2,8450 & 0,9530 & 0,9925 \\
3,1028 & - & 2,6998 & - & 1,0556 & - \\
3,0284 & - & - & - & - & -
\end{tabular}

range was execute the measurements. It would be interesting to accomplish measurement with velocity under the range achieved in this article.

Table 5. Comparison between option CSN and BE

\begin{tabular}{ccccc} 
Sample & Method & $\xi_{c s n}[-]$ & $\xi_{b e}[-]$ & diff [-] \\
\hline \hline \multirow{2}{*}{ Sample1 } & OutRedu & 2,851 & 3,099 & 0,248 \\
& FanRedu & 2,808 & 3,216 & 0,409 \\
\hline \multirow{2}{*}{ Sample2 } & OutRedu & 2,598 & 2,834 & 0,236 \\
& FanRedu & 2,544 & 2,876 & 0,332 \\
\hline \multirow{2}{*}{ Sample3 } & OutRedu & 0,924 & 1,154 & 0,230 \\
& FanRedu & 0,911 & 1,147 & 0,236
\end{tabular}

\section{Conclusion}

In the article were mentioned three ways of measurement of silencers at the suction part. Comparison of friction coefficient calculation by standard ISO 7235 . There were compared three types of measurement with three types of different silencers. Each silencer had diverse construction thus results have broad meaning. In the future should be done measurement on outlet side of the duct and make adequate practice for the effective outcome of measurement.

\section{Acknowledgements}

This work was supported by the Ministry of Education, Youth and Sports of the Czech Republic within the National Sustainability Programme project No. LO1303 (MSMT-7778/2014) and also by the Internal Grant Agency of Tomas Bata University in Zlin under the project No. IGA/CebiaTech/2016/001.

\section{References}

[1] O. Reynolds, Proceedings of the Royal Society of London 35, 24 (1883)

[2] L.F. Moody, Transaction of the A.S.M.E. 66, 14 (1944)

[3] C.F. Colebrook, Journal of the Insitution of Civil Engineers 11, 29 (1939)

[4] T. Von Kármán, L. Edson, The Wind and Beyond: Theodore Von Kármán, Pioneer in Aviation and Pathfinder in Space (Little, Brown, 1967)

[5] P.A. Davidson, Y. Kaneda, K. Moffatt, K.R. Sreenivasan, A voyage through turbulence (Cambridge University Press, 2011)

[6] CSN EN ISO 7235, Standard, Czech office for standards metrology and testing, Prague, CZ (2010)

[7] C.F. Colebrook, C.M. White, Proceedings of the Royal Society of London A: Mathematical, Physical and Engineering Sciences 161, 15 (1937)

[8] S. Genić, I. Arandjelović, P. Kolendić, M. Jarić, N. Budimir, V. Genić, FME Transactions 39, 4 (2011)

[9] CSN EN ISO 5801, Standard, Czech office for standards metrology and testing, Prague, CZ (2009) 